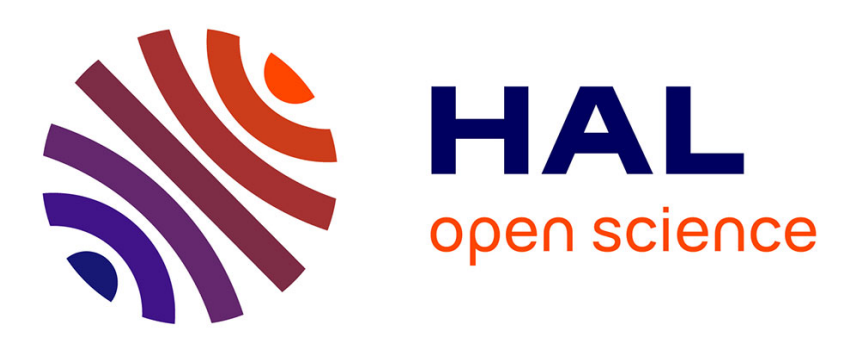

\title{
Scaling laws based metamodels for the selection of the cooling strategy of electromechanical actuators in the early design stages
}

Ion Hazyuk, Marc Budinger, Florian Sanchez, Jean-Charles Maré, Stéphane Colin

\section{To cite this version:}

Ion Hazyuk, Marc Budinger, Florian Sanchez, Jean-Charles Maré, Stéphane Colin. Scaling laws based metamodels for the selection of the cooling strategy of electromechanical actuators in the early design stages. Mechatronics, 2015, 29, pp.67 - 77. 10.1016/j.mechatronics.2015.05.011 . hal-01861798

\section{HAL Id: hal-01861798 \\ https://hal.science/hal-01861798}

Submitted on 25 Aug 2018

HAL is a multi-disciplinary open access archive for the deposit and dissemination of scientific research documents, whether they are published or not. The documents may come from teaching and research institutions in France or abroad, or from public or private research centers.
L'archive ouverte pluridisciplinaire HAL, est destinée au dépôt et à la diffusion de documents scientifiques de niveau recherche, publiés ou non, émanant des établissements d'enseignement et de recherche français ou étrangers, des laboratoires publics ou privés. 


\title{
Cite this article as: I Hazyuk, M Budinger, F Sanchez, J-C Mare, S Colin, Scaling laws based metamodels for the selection of the cooling strategy of electromechanical actuators in the early design stages, Mechatronics in press; doi:10.1016/j.mechatronics.2015.05.011 \\ Scaling laws based metamodels for the selection of the cooling strategy of electromechanical actuators in the early design stages
}

\author{
Ion Hazyuk ${ }^{\mathrm{a}, *}$, Marc Budinger ${ }^{\mathrm{a}}$, Florian Sanchez ${ }^{\mathrm{a}}$, Jean-Charles Maréa ,Stéphane Colin $^{\mathrm{a}}$ \\ ${ }^{a}$ Université de Toulouse, INSA, ICA (Institut Clément Ader), 135, avenue de Rangueil, F-31077 Toulouse, France
}

\begin{abstract}
The integration of electromechanical actuation systems for aerospace applications requires additional thermal stress considerations during the preliminary design, which were not necessary within the hydraulic technology. The favored models during this design phase are simple, continuous and explicit mathematical expressions to enable easy analytical manipulations or implementations in worksheets or optimization loops. The paper shows how such models can be obtained for the desired applications, illustrating the example of a Limited Angle Torque Motor (LATM) with different cooling strategies. The used method for gathering the models is a regression process on data obtained by finite element simulations. The originality of the method stands in the physical insight of the regression function which is based on scaling laws and dimensional analysis. An example of preliminary design of a LATM illustrates the use of the obtained models for the comparison of two possible architectures intended to minimize the mass of the actuator by implementing different cooling strategies.
\end{abstract}

Keywords: natural convection heat transfer coefficient, forced convection, vertical cylinder, design of experiments, motor cooling

\section{Abbreviations}

3D three-dimensional

DDV Direct Drive Valve

DoE Design of Experiments

LATM Limited Angle Torque Motor

RMS Root Mean Square

SLAWMM Scaling-LAW-based MetaModels

\section{Nomenclature}

$\beta \quad$ air thermal expansion coefficient $[1 / K]$

$\Delta \theta \quad$ difference between the winding temperature and bulk temperature of the fluid $[K]$

$\lambda \quad$ fluid thermal conductivity $[\mathrm{W} / \mathrm{mK}]$

$\mu \quad$ fluid dynamic viscosity $[\mathrm{kg} / \mathrm{ms}]$

$\pi_{i} \quad$ dimensionless numbers [-]

$\rho \quad$ fluid density $\left[\mathrm{kg} / \mathrm{m}^{3}\right]$

$\rho_{C u} \quad$ copper density $\left[\mathrm{kg} / \mathrm{m}^{3}\right]$

$\rho_{e l} \quad$ electrical resistivity $[\Omega m]$

$\tau \quad$ copper volume fraction in the copper-resin mixture [-]

$\theta \quad$ temperature $\left[{ }^{\circ} \mathrm{C}\right]$ or $[K]$

$\varphi \quad$ heat flow rate density $\left[W / m^{2}\right]$

${ }^{*}$ Corresponding author. Tel.: +33 (0)5 67048823; fax: $+33(0) 561559950$

Email address: ion .hazyuk@insa-toulouse.fr (Ion Hazyuk)
$B_{r} \quad$ remanent induction of the permanent magnet [T]

$C_{p} \quad$ fluid specific heat capacity $[\mathrm{J} / \mathrm{kgK}]$

$D \quad$ motor diameter $[\mathrm{m}]$

$d_{i} \quad$ geometric dimensions $[m]$

$e \quad$ air gap of the motor $[m]$

$g$ gravitational acceleration $\left[\mathrm{m} / \mathrm{s}^{2}\right]$

Gr Grashof number [-]

$h$ global convective heat transfer coefficient $\left[\mathrm{W} / \mathrm{m}^{2} \mathrm{~K}\right]$

$J \quad$ current density $\left[A / m^{2}\right]$

$L \quad$ motor length $[m]$

$M \quad$ mass $[k g]$

$\mathrm{Nu} \quad$ Nusselt number [-]

Pr Prandtl number [-]

$Q \quad$ heat flow rate $[W]$

Re Reynolds number [-]

$S \quad$ motor external surface $\left[\mathrm{m}^{2}\right]$

$T \quad$ electromagnetic torque $[\mathrm{Nm}]$

$T_{L} \quad$ linear electromagnetic torque $[N]$

$U \quad$ kerosene velocity in the motor air gap $[\mathrm{m} / \mathrm{s}]$

$V_{C u} \quad$ volume of the copper $\left[\mathrm{m}^{3}\right]$

\section{Introduction}

\subsection{The context}

The distribution and the transformation of the electrical energy on-board of embedded systems progressively replace hydraulic 

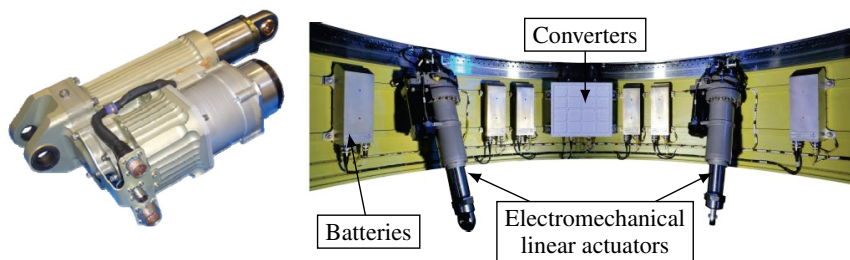

Figure 1: Examples of electrical power transformation in aerospace applications: (left) Spoiler electromechanical actuator on Boeing 787 from MOOG [4], and (right) electromechanical thrust vector control of the Vega launcher [5]

and pneumatic power segments (fig. 1). The more electric aircraft concept illustrates this trend with systems for flight control, braking, engine start, air conditioning, deicing, etc [1]. Aircraft engines can be then optimized while taking advantage of electrical network reliability and ease of maintenance of electrical systems. However, this technology also keeps its own weaknesses, among which are the hard thermal constraints. While within hydraulic technology the hydraulic fluid helps to dissipate the heat generated by the losses, in the case of electric technology the heat remains rather localized. This requires additional, thermal stress, considerations during the design, which may be critical in some cases. For instance, electric motors and their associated converters are often sized on thermal criteria [2]. Electrical network on the aircraft is also strongly thermally constrained, especially if the neighboring airframe is in composite material [3]. Thus, the technological change requires the integration of the new constraints in all the stages of system design.

\subsection{Necessity of simple algebraic models in preliminary design}

As shown in the V-cycle diagram (fig. 2), different types of models are used during the developing process of a mechatronic or more electrical system [6]. In the descending phase of the Vcycle, the system is gradually defined and the detail level of the models increases accordingly. During the specific design stage, the various components of the solution concept are fine-detailed and their three-dimensional (3D) simulation models can be built up. The aim of these $3 \mathrm{D}$ models is the precise evaluation of the developed components by performing local computations (finite element method, etc). Finally, during the integration phase of the process the various components are assembled to form the solution concept and validate its performance. To do this, the highly detailed 3D simulation models are transformed into, less detailed, macro 0D/1D models to perform physical simulations.

Preliminary design is situated on the descending branch of the V-cycle. During this stage, the designer should be able to narrow down to a single candidate solution out of a bunch of possibilities. This process may involve a number of steps such as the choice of one or several candidate concepts/architectures, rough sizing of the components, multi-criteria optimization of the components, comparison of the different solutions and their evaluation with respect to the initial performance requirements. The aim of this stage is to take strategic decisions early in the project, thus avoiding to waste time and money for the detailed design of solutions that in the end may turn to be unfeasible or

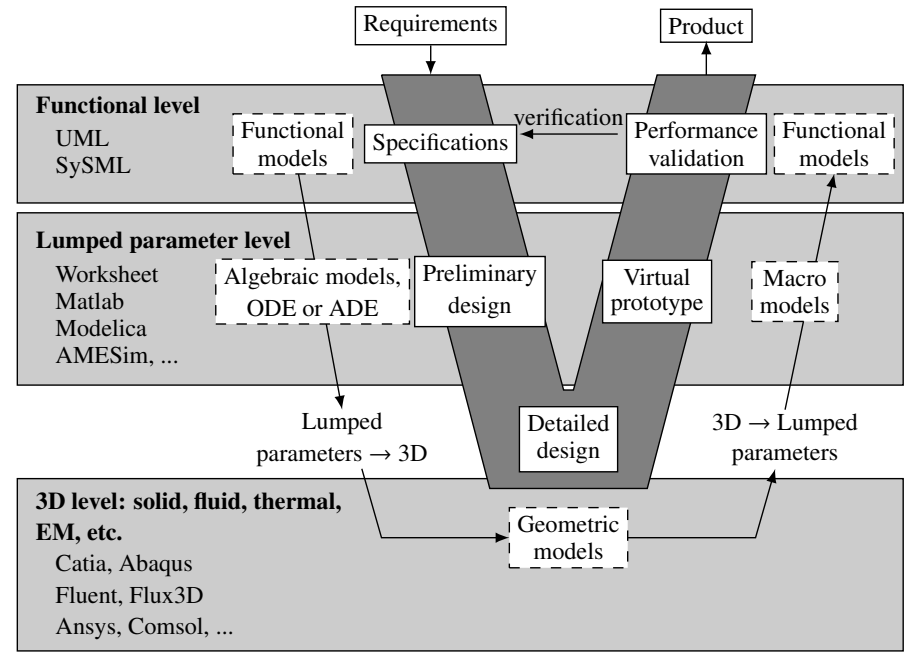

Figure 2: V-cycle diagram for the design of a mechatronic system and its associated modeling

far from optimal. Therefore, the following features are crucial for the models used in this stage:

- simple - to gain rapidity and easy handling, i.e. simple algebraic or ordinary differential equations that can be easily implemented in worksheets or optimization loops;

- explicit - to enable analytic manipulations that provide direct access to the main integration parameters (mainly weight and geometrical parameters) disposing of very little input information (usually functional requirements);

- continuous - at least in the range of conventional parameter variation for the given application, which is especially useful for the optimization.

Since the operation of even a single component may touch on several physical fields, its sizing is often performed within an optimization process. During this process, particular attention is granted to various hard constraints, also called design drivers, because they restrain the space of possible solutions. They alone may eliminate some candidate solution, by this also significantly reducing the optimization task. In the case of electrical power transformation, thermal constraints are frequently among the design drivers [2].

Note that today a wide range of tools are available for the simulation of the thermal behavior, which are based on finite element/volume methods. However, all of them require a precisely defined geometry. Consequently, they can be used in the bottom stage of the V-cycle, or even during the ascending phases via model reduction techniques, but they are totally unsuitable for descending phase, i.e. the preliminary design.

For the early design stage, a preferred approach may be the use of analytic/semi-empirical laws elaborated for basic geometries such as vertical/horizontal plane surfaces [7, 8], cylinders [911], cubes [12] etc. Two aspects may favor this approach. Firstly, at this stage the system component geometries can be replaced by very basic ones such as parallelepipeds and cylinders (see fig. 1 right: cylinders for the motors and parallelepiped for the 
converters). Secondly, these laws can relate some geometrical parameters and physical properties of the component/environment to the global parameters such as thermal flows, temperature differences, heat transfer coefficients, etc. However, the dimensional parameter is usually a single "characteristic length" of the body, which gives models valid for limited ranges of the body aspect ratios. At best, some authors provide tables of coefficients to be used in the formula for different aspect ratios [11]. Moreover, the given models are valid only for particular topological and boundary configurations. This is not advantageous in preliminary design because the dimensions are actually the searched/unknown variables. Then, since the model to be used in preliminary design is not the same for different variation ranges of the searched variable, it is impossible to know beforehand which model has to be used.

Another possibility is to use the same technique that is used during the integration phase of the design process, i.e. model reduction [13]. Here, as shown in fig. 2, the 3D models are transformed in lumped parameter models for the assessment and validation of system specifications. Thus, one can use this knowledge to generate relations between the integration parameters (geometrical dimensions and masses) of interest and the functional parameters (torque, inertia, heat transfer coefficient, etc.). One way to do this is the use of the metamodeling concept $[14,15]$. It supposes to generate a Design of Experiments (DoE) ${ }^{1}$ on a particular case study for which the integration parameters are varied, and then fit a regression model on the obtained data. The problem of the regression models such as polynomial, Gaussian RBF or Kriging is their nonphysical basis which in some cases gives poor predictive capability outside the DoE used for their construction [17]. Nevertheless, a recent paper has shown that if a physical base is used for the regression, the fitting gives simple relations with acceptable predictive capability outside the DoE [17]. This technique is therefore used in this paper.

For the sake of clarity, the basic principles of the used technique for the research of the regression expression are restated in the second section. The third section briefly describes the application for which the models will obtained and tested. The fourth section presents the thermal model construction. Finally, an example of the utilization of the obtained models is given in the fifth section.

\section{Scaling laws, dimensional analysis and metamodels}

This section describes the fundamentals of a particular form of metamodels: the Scaling-LAW-based MetaModels (SLAWMM), which in our opinion are essential for the understanding of the paper body. For an in-depth description of the regression process, the readers are invited to consult [17].

\footnotetext{
${ }^{1}$ Design of experiments is a branch of statistics that deals with the planning of a number of tests to be conducted in order to obtain a good quality models after the regression analysis. Its result is a minimal number of optimally spread configurations for which experiments should be conducted. An in-depth explanation of this tool can be found in [16]
}

\subsection{Scaling laws and dimensional analysis}

Scaling laws based on dimensional analysis, also called similarity laws or allometric models, have been very successfully used throughout the past decades for solving scientific [18] and engineering problems [19-22] and for presenting results in a compact form. The mathematical form of scaling laws is a power law:

$$
y=k L^{a}
$$

where $y$ is the secondary characteristic to be estimated, $L$ the main characteristic length of the component, and $k$ and $a$ are constants. Equation (1) is valid only under material similarity, geometric similarity and uniqueness of the design driver assumptions, as demonstrated in [17] using the Buckingham theorem [23-25]. To illustrate the construction and the use of such laws, a simplified example addressing brushless motors is described hereafter. The objective is to evaluate the mass of the motor as a function of the rated torque.

For magnetic aspects, the torque $T$ can be linked to the main characteristic length and current density through

$$
f(T, \underbrace{L, d_{1}, d_{2}, \ldots, d_{n}}_{n+1}, \underbrace{J, B_{r}}_{m})=0
$$

which, according to the Buckingham theorem, leads to

$$
f^{\prime}(\frac{T}{J B_{r} L^{4}}, \underbrace{\frac{d_{1}}{L}, \frac{d_{2}}{L}, \ldots, \frac{d_{n}}{L}}_{n})=0
$$

The construction of the first dimensionless number $\frac{T}{J B_{r} L^{4}}$ is explained in Appendix B. By Assuming geometrical similarity (the aspect ratio is constant during the size change) the following relation is obtained:

$$
T=k_{1} J L^{4}
$$

where $J$ is the current density, $L$ the length of the motor, $d_{i}$ other geometrical dimensions, $B_{r}$ the remanent induction of the permanent magnet and $T$ the electromagnetic torque. It is assumed here that the main design criterion for the motor is the winding temperature [2]. If convective heat transfer characterized by a constant global heat transfer coefficient $h$ is the dominant thermal phenomenon, the current density $J$ can be linked to the dimensions through:

$$
f(J, \underbrace{L, d_{1}, d_{2}, \ldots, d_{n}}_{n+1}, \underbrace{\rho_{e l}, \theta, h}_{m})=0
$$

which, according to the Buckingham theorem, leads to

$$
f^{\prime}(\frac{\rho_{e l} J^{2} L}{h \theta}, \underbrace{\frac{d_{1}}{L}, \frac{d_{2}}{L}, \ldots, \frac{d_{n}}{L}}_{n})=0
$$

The construction of the first dimensionless number $\frac{\rho_{e l} J^{2} L}{h \theta}$ is explained in Appendix B. Assuming geometrical similarity:

$$
J=k_{2} L^{-0.5}
$$




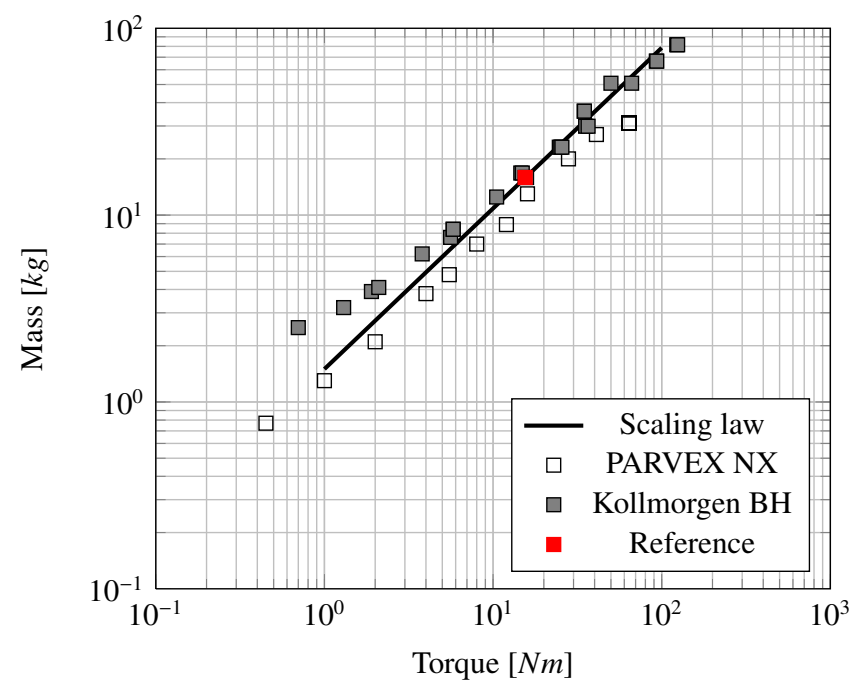

Figure 3: Brushless motor masses according to the nominal torque [26, 27]. The reference point corresponds to the motor whose data where used in the scaling law (eq. (9)) to evaluate the constant $k_{5}$.

where $\rho_{e l}$ the resistivity of the copper and $\theta$ the maximal temperature rise for the winding insulation. Combining these two aspects, modeled by eqs. (4) and (7), one can obtain an estimate of the torque depending on the motor size:

$$
T=k_{3} L^{3.5}
$$

The motor mass can be estimated assuming material and geometrical similarity:

$$
M=k_{4} L^{3}=k_{5} T^{3 / 3.5}
$$

Figure 3 compares the eq. (9) with data from industrial catalogs. It shows that scaling laws can provide good trend for the quantities of interest for such components.

\subsection{Scaling-LAW-based MetaModels (SLAWMM)}

To maintain the physical meaning and the benefits of scaling laws, the regression model will be based on the power form from eq. (1). However, to eliminate the assumptions of geometric similarity and uniqueness of design driver, estimation models of the following, more general form will be sought:

$$
y=f\left(L, \pi_{1}, \pi_{2}, \ldots,\right)=k\left(\pi_{1}, \pi_{2}, \ldots\right) L^{a\left(\pi_{1}, \pi_{2}, \ldots\right)} \text { with } \pi_{i}=\frac{d_{i}}{L}
$$

where $y$ is the parameter to be estimated, $L$ is the characteristic length of the component, $d_{i}$ are the secondary geometrical dimensions of the component and $\pi_{i}$ are the dimensionless numbers representing the aspect ratios.

With geometrical similarity, the aspect ratios are constant $\left(\pi_{i}=C^{s t}\right)$ and the form from eq. (10) simplifies into the classic scaling law form, eq. (1). The desired form of the model (10) lends itself to direct regression less easily than polynomial response surfaces [28], product of pure power laws, radial basis functions $[29,30]$, or kriging $[31,32]$ directly on the parameter $y$. Budinger et al. [17] proposed an approach to determine the shapes of functions $k\left(\pi_{1}, \pi_{2}, \ldots\right)$ and $a\left(\pi_{1}, \pi_{2}, \ldots\right)$ representative of multiplicative and power coefficients.

Budinger et al. [17] tried to find an expression linking a variable of interest to the main dimensions of a component. Therefore, they defined and used only dimensionless numbers that represent aspect ratios. The present paper, however, attempts to express the variable of interest which depends not only on geometrical dimensions but also on other physical variables and/or properties of the used materials. Therefore, the dimensionless numbers that will be used later in the paper will also include physical properties.

\section{Description of the case study}

\subsection{Electromechanical actuator and its considered cooling strategies}

Aircraft flight control surfaces such as ailerons, elevators, flaps, spoilers, etc. are manipulated by actuation systems. They generally employ electric motors somewhere in the power transmission chain. For instance, brushless motors can be used at various levels of the system such as :

- In compressors to generate the hydraulic power for hydromechanical actuators.

- In electro-mechanical actuators to drive the screw-nut system.

- In Direct Drive Valves (DDVs) to control hydrostatic actuators, etc.

This paper focuses on the last type of applications where the used motor is a LATM $[33,34]$. An example of such apparatus is illustrated in fig. 4. For the selection of a suitable motor, two main aspects should be considered: (i) the motor must be able to deliver a certain nominal torque, $T$, and (ii) since the DDV is itself a closed loop system, it must ensure a given bandwidth. In order to achieve the latter, the motor mechanical inertia should be carefully chosen. Actually, the time constant of the LATM dynamical model depends mainly on its mechanical inertia (more exactly it is the ratio between the inertia and the viscous friction). Thus, if its corresponding cutoff frequency (the inverse of the time constant) is smaller than the required closed loop bandwidth, the controller should have larger gains. This will give higher amplitudes for the command which, at best, will lead to larger energy consumption. At worst, the maximal torque of the motor will be smaller than the maximal command (the motor torque will enter in saturation) and the system will not ensure the required closed loop bandwidth.

The nominal torque of the LATM depends on its size and the maximal electrical current density. The maximal current density, in turn, is limited by the maximum winding temperature that prevents the insulation degradation. In other words, the nominal torque of the motor is that one which avoids the overheating of its windings. For ground-based applications, the nominal torque of a motor, provided by constructor catalogs, is generally calculated for an open space at a fixed environmental temperature, usually $25^{\circ} \mathrm{C}$ or $40^{\circ} \mathrm{C}$. In aerospace applications 


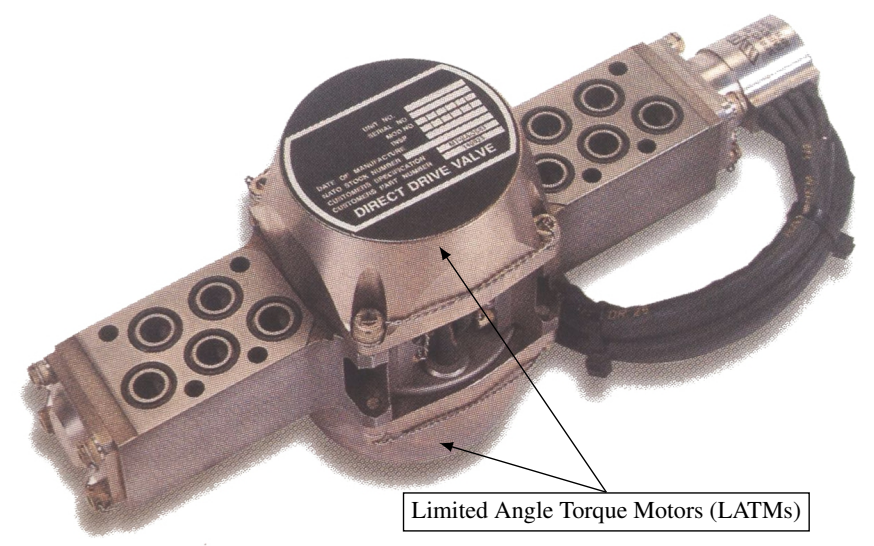

Figure 4: Illustration of a DDV driven by a LATM [35]

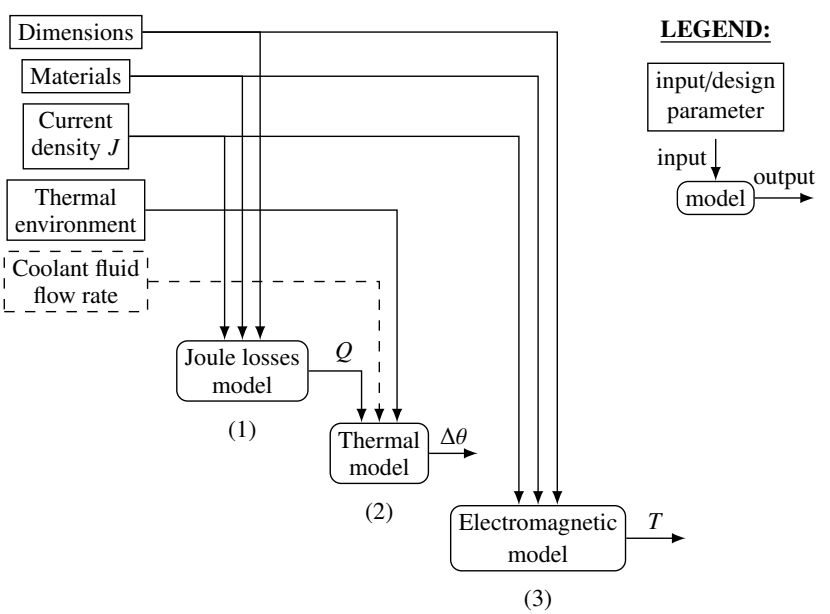

Figure 5: N-squared diagram of the LATM modeling process the thermal and mechanical boundary conditions may differ significantly, which make catalog data unsuitable. Furthermore, different cooling strategies may be employed in order to improve the thermal behavior of a motor. The usually accepted cooling strategies in this area on which engineers may count during preliminary design are natural and forced convection. Heat removal by conduction toward the structure via the mounting brackets is generally prohibited. Thus, heat may be removed from the windings either by natural convection via its housing (or via a heat sink), and when this is not efficient enough (or the needed heat sink is too heavy) heat may be removed by forced convection via a fluid circulating through its air gap. In both cases, the estimation of the average heat transfer coefficient is crucial for the preliminary design stage. Moreover, since the mechanical inertia of the motor depends on its geometry, implicitly it is impacted by the cooling capabilities of the motor.

The particularity of this application is that it is used for combustion control of a helicopter turboreactor. Thus, since kerosene is available nearby, if necessary, it may be used for equipment cooling.

\subsection{Modeling approach}

As stated in the previous paragraph, the nominal torque delivered by a LATM is limited by the hot-spot temperature of its windings. Thus, to assist the engineer in the selection of a LATM with an appropriate cooling strategy, one would need one or several expressions relating the nominal torque of the motor to the hot-spot temperature of its windings for a given size and cooling strategy. Hence the need of reduced models to predict the electro-magnetic and thermal phenomena taking place in the motor during its operation. This task is achieved in three steps, as shown in fig. 5. First, electric Joule losses, $Q$, are evaluated from motor geometry, materials and current density. Actually, in electrical motors there are other sources of heat generation such as mechanical friction and iron losses (hysteresis, Foucault current). However, since LATMs function at small average velocities (common for servo-positioning actuators), these ones are usually low in comparison with electric Joule losses and thus can be neglected. Secondly, a model is established linking the winding temperature rise above the environmental temperature, $\Delta \theta$, and the input parameters with the corresponding losses $Q$. When only natural convection is involved, the environment represents the ambient air while in the case of forced convection, the environment is considered to be the temperature of the cooling fluid. In the latter case, the coolant flow rate is also an input of the thermal model. The output of this model helps to determine if the current density $J$ avoids winding overheating. Lastly, a third model is established to relate the torque to the motor geometry, materials and current density.

\section{Model construction and validation}

Figure 6 illustrates the sectional views of the studied LATM. Finite element simulations were performed in COMSOL Multiphysics. For each thermal simulation, the hot-spot temperature of the motor windings was retrieved in order to use it for the regression process. Similarly, the torque was retrieved from electromagnetic simulations.

\subsection{Electromagnetic modeling}

As stated in the previous section, the torque of a motor depends on its geometry, material properties and current density:

$$
f\left(T_{L}, D, J, B_{r}\right)=0
$$

where $T_{L}$ is the linear torque, $D$ the motor diameter, $J$ the current density and $B_{r}$ the magnet remanent induction. In the case of LATM geometry, the torque is very little influenced by the magnetic circuit saturation under the effect of the current density $J$. According to the Buckingham $\pi$ theorem, the problem can be expressed as:

$$
f^{\prime}\left(\pi_{1}\right)=0
$$

where $\pi_{1}=\frac{T_{L}}{J B_{r} D^{3}}$, which implies that the motor's electromagnetic torque can be expressed as:

$$
T_{L}=k J B_{r} D^{3}
$$




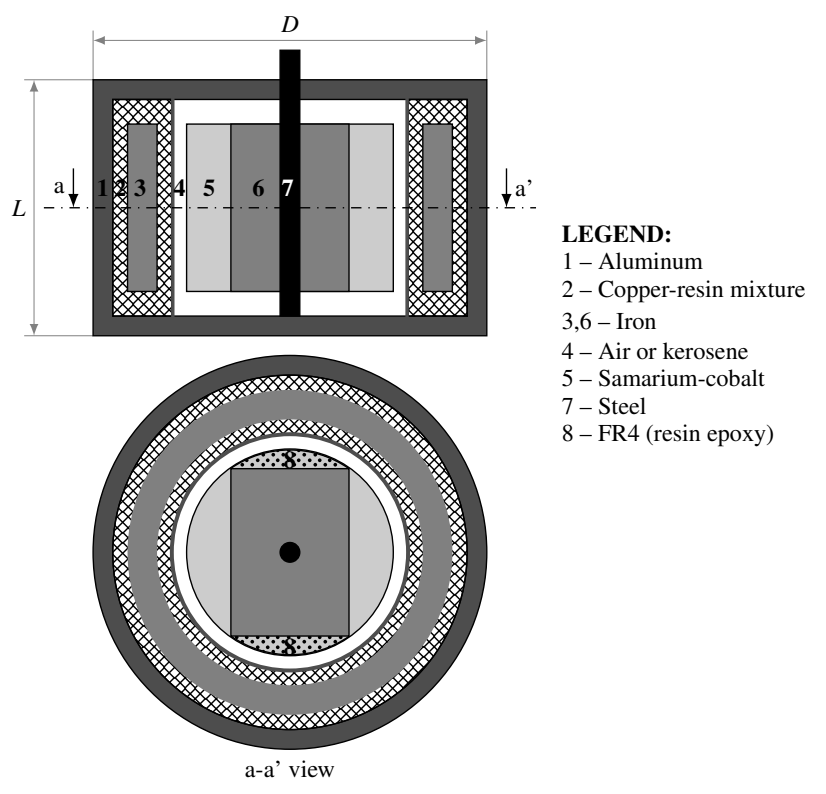

Figure 6: Sectional views of the studied LATM

In order to obtain the parameter $k$ by regression, finite element simulation were carried out in Comsol. Since we measured the linear torque, a planar electromagnetic model was used (fig. 6 bottom) where the air gap $e$ varied with the same ratio as the motor diameter $D$ (this is an observation that can be made on the Parvex motors range [26]) and the remanent induction of the magnet was constant $\left(B_{r}=1 T\right)$. The mesh contained only triangular elements and its independence on the solution has been tested. During the motor size variation, the computational domain always consisted of about 30000 elements.

The value of the coefficient $k$ in eq. (13) is obtained by regression on simulation data $(k=0.00418)$. These simulation data were obtained for a five level full factorial DoE on the current density $J$ and motor diameter $D$. The variation range of the current density $J$ was $10^{6}-10^{7} \mathrm{~A} / \mathrm{m}^{2}$ and that of the diameter is given in table 1. Figure 7 compares the electromagnetic torque gathered from simulations with that obtained by eq. (13) for two data sets (first set - "DoE" and second set - "Outside DoE" ranges, indicated in table 1); the first set is used for regression and the second set is generated only for validation purpose. As it may be observed, eq. (13) predicts the torque based on motor's geometry and current density with satisfactory precision.

Similarly, the losses transformed in heat by Joule effect, are estimated by:

$$
Q=\rho_{e l} \tau J^{2}
$$

where $Q$ is the heat generation rate, $\rho_{e l}$ the electrical resistivity of the copper and $\tau$ the copper volume fraction in the copper-resin mixture ( $\tau=0.4$ for all the sizes).

\subsection{Natural convection modeling}

A previous study [17] have shown that if the windings of the LATM are flooded in potting resin, the thermal gradient appears essentially in the convective boundary layer. For usual configurations, the ratio between the convective $\left(R_{c v}\right)$ and conductive

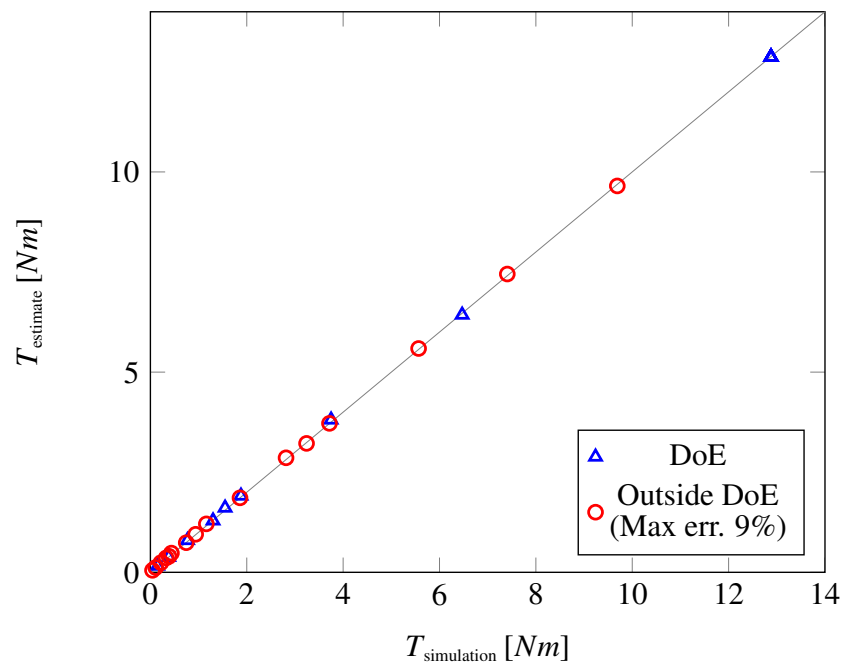

Figure 7: Comparison of the electromagnetic torques obtained by Comsol simulation, $T_{\text {simulation }}$ and eq. (13), $T_{\text {estimate }}$

Table 1: Range of parameter variations for suspended cylinder and LATM study

\begin{tabular}{lcc}
\hline Variable & $\begin{array}{c}\text { DoE range } \\
\text { (for regression) }\end{array}$ & $\begin{array}{c}\text { Outside DoE range } \\
\text { (for model validation) }\end{array}$ \\
\hline$L(\mathrm{~cm})$ & $2-6$ & $2-10$ \\
$D(\mathrm{~cm})$ & $2.5-10$ & $2.5-12.5$ \\
$\pi_{2}$ & $0.8-2$ & $0.8-8$ \\
$G r$ & $2 \cdot 10^{6}-4 \cdot 10^{6}$ & $2.7 \cdot 10^{4}-6 \cdot 10^{8}$ \\
$R e$ & $12.9-25.8$ & $5-50$ \\
\hline
\end{tabular}

$R_{c d}$ thermal resistances is very high: $R_{c v} / R_{c d} \approx 15$. Thus, the temperature of the motor body is practically homogeneous. Consequently, it is acceptable to consider that the heat exchange can be characterized only by its convection heat transfer coefficient.

In day-to-day applications, the actuators are attached to a frame or to another component. In the studied application case, it is considered that the LATM is vertically placed on a horizontal flat surface. For the study of the worst case scenario, it is considered that there is no heat exchange between the LATM and the support surface, i.e. adiabatic boundary conditions are assumed on the bottom face of the motor. This is often imposed by the system engineer. If, for a different application, this assumption is invalid, an additional study must be conducted similarly to the one for the forced convection which is described in the next section.

For this study, the finite element model built in Comsol is axisymmetric (fig. 6 top). The simulated motor is placed in a confined space, whose size is ten times larger than the cylinder itself in order to eliminate the impact of confinement degree on the hot-spot temperature. The boundary conditions are a constant heat flux in the LATM windings and a bulk air temperature of $300 \mathrm{~K}$. The following assumptions are considered for modeling and simulation:

- The air flow around the motor is assumed laminar;

- Given the considered range of the environment temperature, the fluid properties are also assumed to be constant. 
Actually, when the density variation is small, the Boussinesq approximation can be made. It assumes that the density is constant everywhere in the equations except in the buoyancy term itself. For the latter it is considered that $\rho-\rho_{a}=-\rho_{a} \beta\left(\theta-\theta_{a}\right)$, where $\rho$ and $\theta$ are the density and the temperature in the buoyancy term, $\rho_{a}$ and $\theta_{a}$ are the ambient density and temperature respectively and $\beta$ is the air thermal expansion coefficient [36];

- The copper-resin mixture composing the LATM windings is homogeneous with a copper volume fraction of $\tau=40 \%$. Mixture properties are calculated as suggested by [37];

- Ideal solid-solid contacts are considered in the LATM. Thermal contact resistances are thus neglected;

- At this stage, only convective heat transfer is considered. The radiative heat transfer is considered to be decoupled and thus modeled by the Stefan-Boltzmann law. This assumption was verified and validated by additional simulations.

The mesh in the solid parts contained triangular elements and in the fluid part contained rectangular elements. Mesh independence on the solution has been tested.

The studied problem depends on eight dimensional variables:

$$
f\left(\rho, \mu, \lambda, C_{p}, g \beta \Delta \theta, L, D, h\right)=0
$$

where $\rho$ is the air density, $\mu$ is the air dynamic viscosity, $\lambda$ is the air thermal conductivity, $C_{p}$ is the air heat capacity, $g$ is the gravitational acceleration, $\Delta \theta$ is the winding temperature rise above the bulk air temperature, $\beta$ is the air thermal expansion coefficient, $L$ is the LATM length, $D$ is the LATM diameter and $h$ is the global convective heat transfer coefficient. The combination $g \beta \Delta \theta$ corresponds to the Archimedes force in Boussinesq approximation. Since the problem depends on eight dimensional variables and four independent fundamental quantities (mass, distance, time and temperature), according to the Buckingham theorem the problem can be reformulated in terms of four dimensionless variables:

$$
N u=f^{\prime}\left(\pi_{2}, P r, G r\right)
$$

where $\pi_{2}=\frac{D}{L}$ is the aspect ratio, $G r=\frac{g \beta \varphi \rho^{2} L^{4}}{\mu^{2} \lambda}$ Grashof number, $N u=\frac{h L}{\lambda}$ Nusselt number and $\operatorname{Pr}=\frac{\lambda}{\mu C_{p}}$ Prandtl number. Since in this application the convection is generated by the heat produced in the cylinder, the Grashof number is defined in terms of the heat flow rate density $\varphi$ instead of temperature difference $\Delta \theta$ (via the Fourier's law). For the given parametric study, the Prandtl number is constant. Thus, according to [17], the researched mathematical form of the convective heat transfer coefficient is:

$$
h=k\left(\pi_{2}, G r\right) L^{a\left(\pi_{2}, G r\right)}
$$

By replacing the expression of the Nusselt number in eq. (16) gives:

$$
N u=\frac{h l}{\lambda}=f^{\prime}\left(\pi_{2}, G r\right)
$$

or

$$
h=f^{\prime}\left(\pi_{2}, G r\right) \lambda L^{-1}
$$
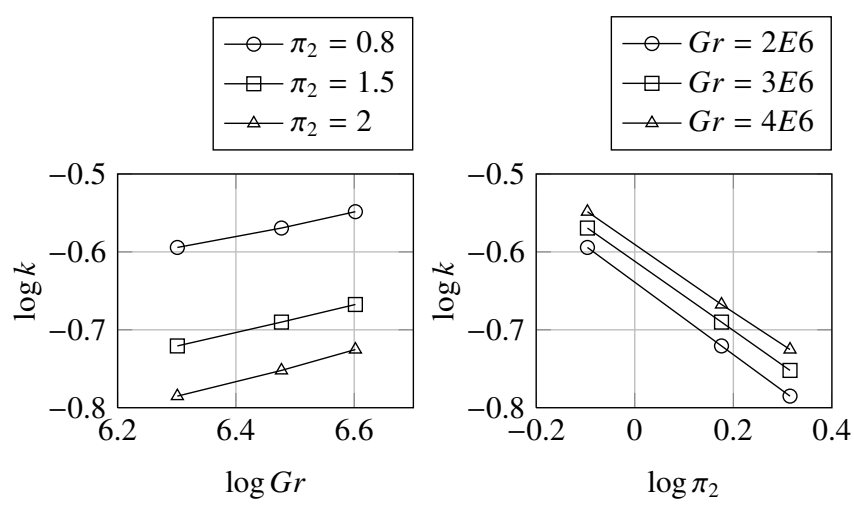

Figure 8: Plots in logarithmic bases of the multiplicative function $k$ versus Grashof number (left) and form factor $\left(\pi_{2}\right)$ (right)

Thus, the power expression is $a\left(\pi_{2}, G r\right)=-1$ while $k\left(\pi_{2}, G r\right)=$ $f^{\prime}\left(\pi_{2}, G r\right)$ should be identified.

The regression process is performed in two steps. First, the mathematical form of the multiplicative expression $k\left(\pi_{2}, G r\right)$ is identified and then the regression itself is performed on the identified bases. To proceed for the regression, a three level full factorial DoE was generated in logarithmic scale, whose parameters range of variation are given in table 1 in the second column. Using this DoE, the data necessary for the regression process were obtained by finite element simulations.

Following the methodology described in [17], the variation of the multiplicative coefficient $k$ versus $\pi_{2}$ while $G r$ is constant is plotted on a logarithmic base in fig. 8 left. The same is done for the second dimensionless number $G r$ in fig. 8 right. Thus fig. 8 shows that the expression of the multiplicative coefficient can be expressed as a power law: $k\left(\pi_{2}, G r\right)=A \pi^{B} G r^{C}$. By performing a nonlinear regression on the simulation data $(\mathrm{DoE}-$ table 1 second column), the obtained coefficients $A, B$ and $C$ are:

$$
h_{\text {SLAWMM }}=0.61 \pi_{2}^{-0.179} G r^{0.19} \lambda L^{-1}
$$

The results obtained by applying the SLAWMM method are compared with those obtained by two other correlations provided by the literature for a representative geometrical shape. The considered geometry is a suspended vertical cylinder since this shape resembles most to the LATM geometry. Moreover, the same procedure is used by motor manufacturers to study the thermal behavior of their motors [38]. The first compared correlation, suggested by Minkowycz and Sparrow [10], evaluates the heat transfer coefficient by an analytical study of the boundary layer for different sizes and thermal boundary conditions. The second one, presented in the Handbook of Heat Transfer [11], gives an experimental correlation of the convective heat transfer coefficient for a suspended vertical cylinder. Figure 9 compares the convective heat transfer coefficients evaluated by SLAWMM, Minkowycz and Handbook methods. Two data sets were used for the SLAWMM method; one set was used for the regression procedure, and another data set is used for the evaluation of prediction capabilities of the correlation. For the second data set, the variation ranges of the parameters exceed those of the DoE used for regression (refer to the corresponding column 


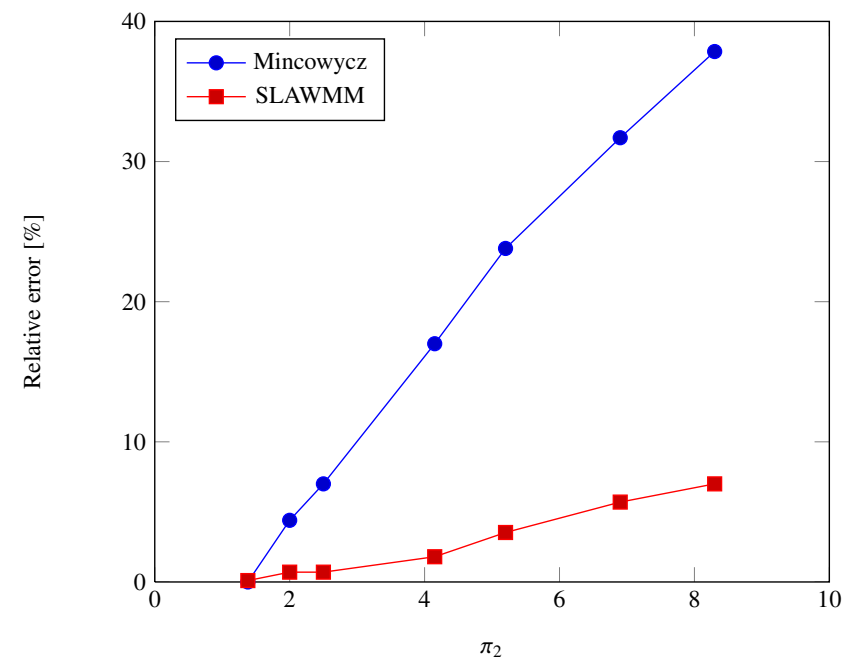

Figure 10: Impact of the aspect ratio on estimation precision of Minkowycz and SLAWMM methods

in table 1). By analyzing the results obtained in fig. 9 it can be concluded that Handbook method does not offer sufficiently accurate predictions for parameter variations in the range given in table 1 . Minkowycz method offers satisfactory prediction capabilities, except for some particular cases where the precision may be unacceptable (see fig. 9 left, circled areas). These values are obtained for $\pi_{2}>2$, that is to say the motor has a large diameter as compared to its height. In practice, such geometry may be particularly interesting when lightweight actuators with large torque are required (see eq. (13)). Actually, Minkowycz method is based on the analytic resolution of the convective boundary layer of the lateral surface of the cylinder. Thus, when motor diameter exceeds its height, convection on the top surface of the motor significantly impacts the overall mean heat transfer coefficient. As shown in fig. 10, the larger the aspect ratio $\pi_{2}$, the higher the relative error. By contrast, in the SLAWMM method the correlation is obtained by regression on simulation data, so the impact of the top surface convection is still captured. Therefore, eq. (24) offers better prediction capabilities as compared to Minkowycz method (see fig. 10). Furthermore, in the case of SLAWMM method, the prediction error can be decreased even more by extending the DoE over the regions of interest, while this is impossible to achieve within the Minkowycz method.

The importance of a simple mathematical form of the obtained model in eq. (20) lays in its further use for preliminary design. During this phase, the modeling process described in fig. 5 is reversed; engineers tend to impose the maximal temperature rise in the motor winding and calculate the maximal admissible current density, which is then used for torque calculation. The current density is obtained from the Newton law, where the heat transfer coefficient should be used. Thus, since the heat transfer coefficient in eq. (20) depends on the heat flow rate (via Grashof number), eq. (20) cannot be used directly in this form. Nevertheless, after some simple mathematical manipulations, described in Appendix A, one is enable to express this coefficient in terms of motor geometry and winding temperature rise above

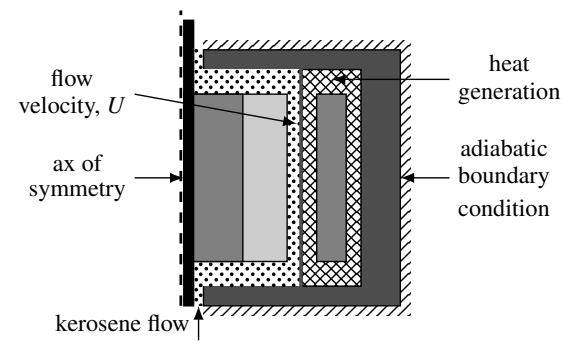

Figure 11: Simulation model for the LATM in the case of internal cooling

the environmental temperature, as in eq. (21). It should be noted that if the mathematical form of eq. (20) would not be so simple, probably it was more difficult, or even impossible, to obtain eq. (21).

$$
h_{\text {SLAWMM }}=1.05 D^{-0.22} L^{-0.0753} \Delta \theta^{0.234}
$$

\subsection{Internal forced convection modeling}

For this case study the same LATM is considered except for the fact that a fluid passes through the air gap of the motor to cool it form inside. The used fluid is kerosene - the same one used by the hydraulic jack (for certain applications in the proximity of the reactors). Several simulation tests revealed that even for the smallest flow rates usually encountered in this area of applications, the heat evacuated by natural convection toward the ambiance is insignificant as compared to the heat evacuated by the coolant fluid. Moreover, in the range of the studied geometrical dimensions and for the usually encountered flow rates, the hydraulic and thermal entry lengths are small (max. 10\%) as compared to the size of the duct $(L)$. Therefore, it can be considered that the flow is laminar and fully developed thermally and hydrodynamically. Therefore, for this configuration, adiabatic limit condition is assumed on the external surface of the LATM (see fig. 11). Given all these assumptions, the problem depends on nine dimensional variables:

$$
f\left(\rho, \mu, \lambda, C_{p}, U, L, D, e, h\right)=0
$$

where $\rho$ - kerosene density, $\mu$ - kerosene dynamic viscosity, $\lambda$ - kerosene thermal conductivity, $C_{p}$ - kerosene heat capacity, $U$ - kerosene velocity along the lateral surface of the LATM, $L$ - cylinder's length, $D$ - cylinder's diameter, $e-$ the air gap and $h$-convective heat transfer coefficient. Since the problem depends on nine dimensional variables and four independent fundamental quantities, it can be reformulated in terms of five dimensionless variables:

$$
N u=f^{\prime}\left(\pi_{2}, \pi_{3}, \operatorname{Pr}, R e\right)
$$

where $\pi_{2}=\frac{D}{L}$ and $\pi_{3}=\frac{e}{D}$ aspect ratio, $N u$ and $\operatorname{Pr}$ Nusselt and Prandtl numbers defined as previously and $R e=\frac{\rho U e}{\mu}$ the Reynolds number. Since the air gap of the motor varies with the same rate as the diameter, the form factor $\pi_{3}$ is constant and thus it can be omitted from the analysis. Assuming small variations of the fluid temperature, the same applies to the Prandtl number. The dimensional analysis which gave these dimensionless 

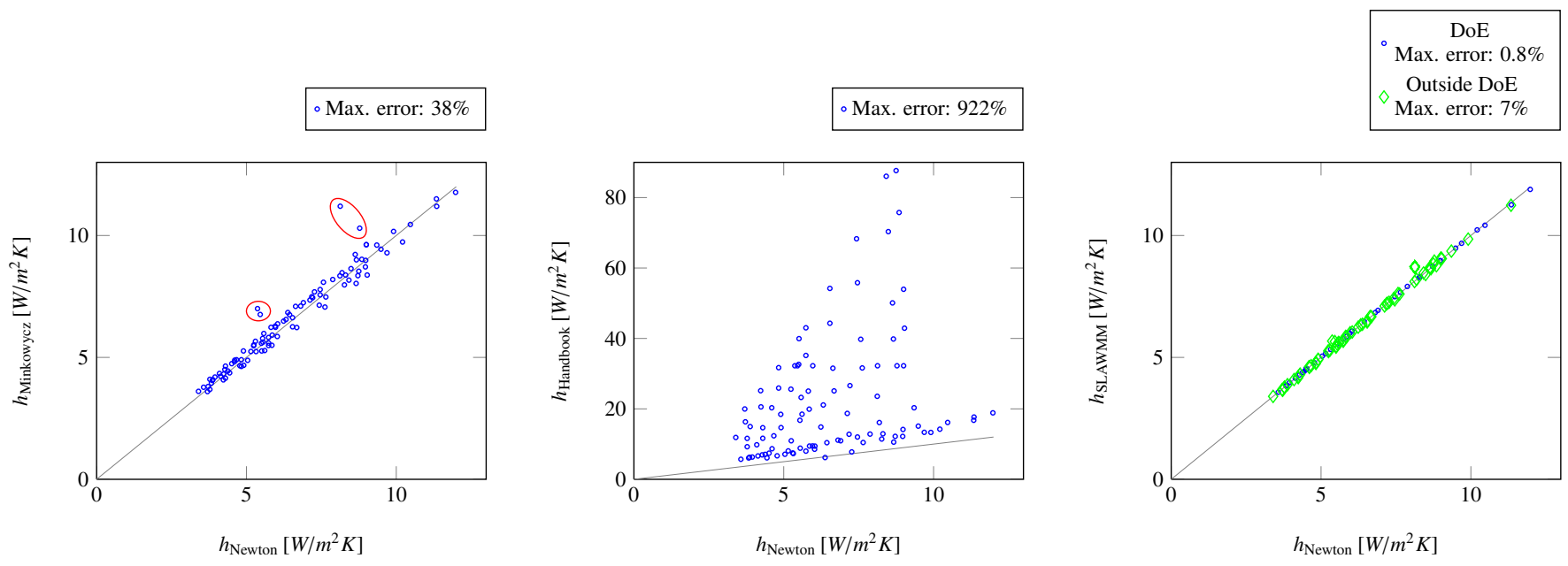

Figure 9: Comparison of the convective heat transfer coefficient for the LATM using Minkowycz (left) Handbook (center) and SLAWMM (right) methods

numbers, is conducted by considering only the heat exchange between the lateral surface of the motor with the fluid. The heat exchanged between the fluid and the top/bottom surfaces of the motor are neglected at this stage. According to SLAWMM methodology the researched mathematical shape of the heat transfer coefficient is:

$$
h=k\left(\pi_{2}, R e\right) L^{a\left(\pi_{2}, R e\right)}
$$

For the same reasoning as in the previous, natural convection, case the power expression is $a\left(\pi_{2}, R e\right)=-1$, which gives:

$$
h=k\left(\pi_{2}, \operatorname{Re}\right) \lambda L^{-1}
$$

Applying [17], the variation of the multiplicative coefficient $k$ versus one dimensionless number while the other one is constant, is plotted in fig. 12. In this case, its mathematical form is a polynomial:

$$
k\left(\pi_{2}, R e\right)=A_{0}+A_{1} \pi_{2}+A_{2} \pi_{2}^{2}+B_{1} R e+B_{2} R e^{2}+C \pi_{2} R e
$$

By performing the regression itself, the obtained coefficients are:

$$
\begin{array}{r}
h_{\text {SLAWMM }}=\left(77-42.795 \pi_{2}+2.487 \pi_{2}^{2}+5.876 R e-0.070 R e^{2}-\right. \\
\left.-0.295 \pi_{2} R e\right) \lambda L^{-1}
\end{array}
$$

Figure 13 compares the convective heat transfer coefficient obtained by eq. (27) with the one obtained by the Newton law, for two data sets. The first data set was used for the regression itself (DoE) and the second one is used for validation purpose (outside the DoE). It can be noted that there is a group of estimates (circled points in fig. 13) that are quite far from the simulated ones. This group is obtained for geometries with $\pi_{2}=2.6$. When the aspect ratio becomes larger, the estimation error increases even more. Since the dimensional analysis was conducted for the heat exchange between the lateral surface of the motor and the fluid, this explains the validity limits of the obtained correlation. That is to say, once the motor diameter is larger than its high, the heat transferred by the top and bottom surfaces becomes significant.

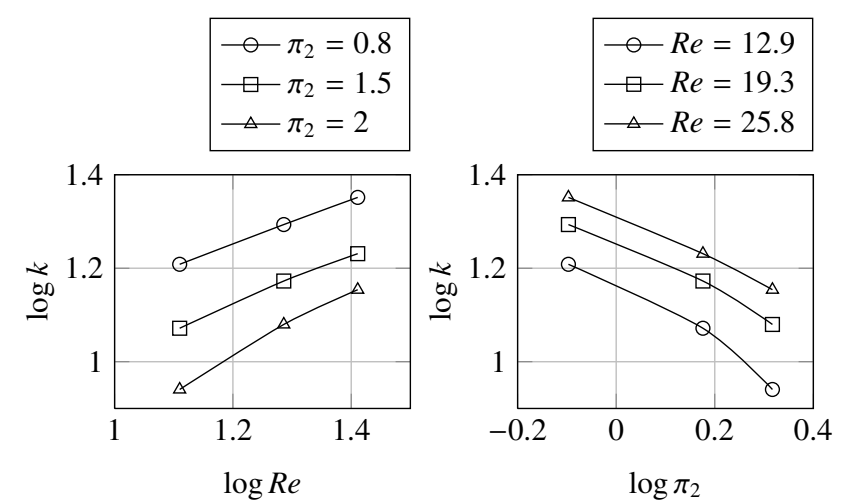

Figure 12: Plots in logarithmic bases of the multiplicative function $k$ versus Reynolds number (left) and form factor $\left(\pi_{2}\right)$ (right)

In order to extend the validity range of the model for larger aspect ratios, the authors presume that the dimensional analysis should also consider the heat exchange via the top/bottom surfaces of the motor. This will give additional dimensionless numbers that potentially may help to minimize the estimation error for the usage beyond the DoE used during the regression.

\section{Metamodel use for the selection of the cooling strategy}

This section presents an example of the utilization of previously obtained metamodels in preliminary design of a LATM with its cooling strategy intended for the command of a DDV in aerospace applications. The usual requirements for such applications are the peak and/or Root Mean Square (RMS) torques, the maximal stroke and the actuator bandwidth. Given the application area, the mass of the actuator is also regarded. The peak torque of a permanent magnet motor is usually limited by the demagnetization, its RMS torque is limited by the overheating of the winding insulation [39] and its bandwidth - mainly by the rotor mechanical inertia. Here, the RMS torque is considered to be the design driver because of the severe thermal environment (next to the reactor), while the mass and the inertia are surveyed 


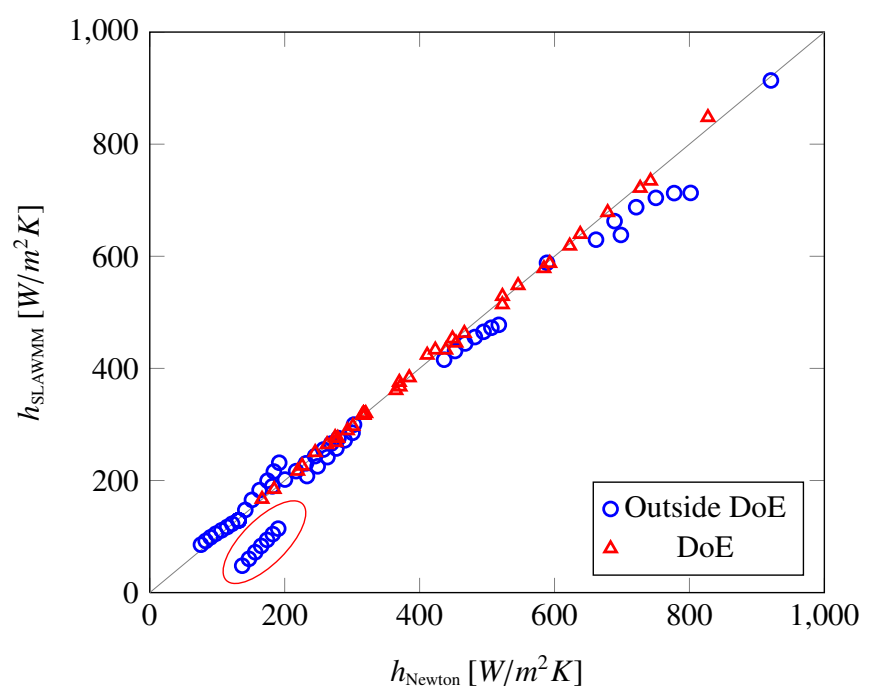

Figure 13: Comparison of the convective heat coefficient for the internal forced convection

to remain below the acceptable limits. Thus, for the application the following input data and objectives are considered:

- the required RMS torque is $0.1 \mathrm{Nm}$

- the maximal winding temperature is $140^{\circ} \mathrm{C}$

- the environment temperature is $90{ }^{\circ} \mathrm{C}$

- the LATM mass $M$ should be smaller than $200-250 g$

- the LATM inertia I should be smaller than $10^{-6} \mathrm{~kg} \mathrm{~m}^{2}$

For the estimation of the mass and inertia of the LATM, the following scaling laws are used [40]:

$$
\begin{gathered}
M=k D^{2} L \\
I=k D^{4} L
\end{gathered}
$$

These laws can be used for two distinct cases:

- To check if a given motor will resist overheating in a different thermal environment from than which is specified in the catalog

- To find the dimensions of a motor for a given list of specification (torque, thermal constraints, cooling, etc.) within an optimization loop.

The sizing procedure of the LATM can be resumed in four steps, fig. 14:

1. Heat transfer coefficient calculation: the radiative and convective (natural or forced) heat transfer coefficients are calculated for a given motor dimension ( $D$ and $L$, chosen by the user or the optimization algorithm) and a maximal allowed temperature rise in the motor's winding.

2. Thermal losses calculation: the maximal heat flux density is calculated using the Newton law which gives the corresponding current density for the LATM.

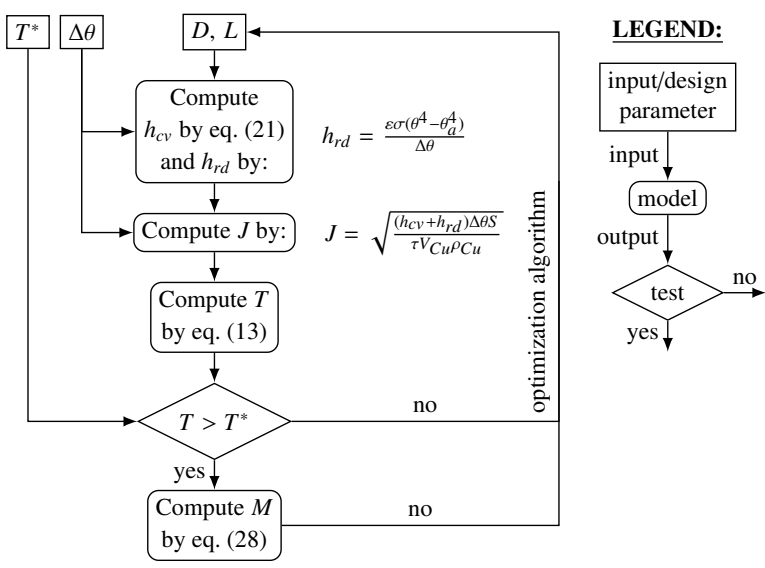

Figure 14: Preliminary LATM sizing by optimization and using the constructed metamodels. In the optimization problem, $T>T^{*}$ represents the inequality constraint and the eq. (28) represents the objective function to be minimized

3. Torque calculation: the RMS torque of the LATM is calculated using the previously obtained scaling law. If the obtained torque is lower than the required one, the optimization repeats steps $1-3$.

4. Mass and inertia calculation: the motor's weight and/or rotor's inertia are calculated, and according to the optimization criteria the steps 1-4 may be repeated or not.

During the first try, with the LATM cooled by natural convection, when the LATM mass was optimized, the sizing gave a motor with the following characteristics: $D=5.56 \mathrm{~cm}, L=2 \mathrm{~cm}$, $m=252 \mathrm{~g}$ and $I=9.58 \cdot 10^{-6} \mathrm{~kg} \mathrm{~m}^{2}$. The obtained mass is acceptable but the inertia is too large. A second try, where the inertia of the LATM rotor was optimized gave $D=2.84 \mathrm{~cm}$, $L=15 \mathrm{~cm}, m=492 \mathrm{~g}$ and $I=4.9 \cdot 10^{-6} \mathrm{~kg} \mathrm{~m}^{2}$. The rotor's inertia is smaller but still too important while the motor is too heavy and its aspect ratio $D / L$ is unrealistic $(\pi=0.16)$. Thus, a solution to minimize the motor's mass and inertia is to change the cooling strategy by passing a fluid in the air gap (internal forced convection). The considered fluid is kerosene at $90{ }^{\circ} \mathrm{C}$ and a flow rate of $3 \cdot 10^{-3} \mathrm{l} / \mathrm{s}$. The minimization of the mass gave $D=2.9 \mathrm{~cm}, L=2 \mathrm{~cm}, m=68 \mathrm{~g}$ and $I=0.7 \cdot 10^{-6} \mathrm{~kg} \mathrm{~m}^{2}$. In this case all the design requirements are fulfilled.

Even if a motor with exact sizes obtained in the last example does not exist in catalogs, the aim is to give to the designer a rough idea about the achievable performances when different cooling architectures are used. For this example, the designer can see that by changing the cooling strategy, the motor can be more than three times lighter and its inertia can be about ten times smaller.

\section{Conclusions}

This paper attempts to apply the technique of scaling laws based metamodel construction to obtain thermal models adapted for preliminary design of electromechanical actuation systems. An adapted model for preliminary design should relate the integration variables to the other variables of interest via simple, 
explicit and continuous expressions. Given that during preliminary design the geometry of the components may be viewed as basic geometrical shapes (rectangles and cylinders), some existing semi-empirical models were tested. Although these models gave acceptable estimations for boundary conditions where they were defined, the results may diverge for slightly different topological configurations. For instance, the expression for the estimation of the convective heat transfer coefficient, applicable to a suspended cylinder, don't give accurate estimations for a cylinder, which is three times larger than its high, sitting on an adiabatic surface. Moreover, the mathematical expressions of the semi-empirical models are often laborious which may burden their integration in worksheets or optimization loops (although from scientific point of view this is not an issue, it may count in industrial environment).

The method presented in the paper is based on scaling laws metamodeling, which by their physical fundamentals provide simple mathematical functions while presenting interesting extrapolation capacities. Moreover, the advantage of this method is that it enables to adapt the researched model to a topology requested by the application, for which no anterior studies are available. In comparison with the previous study of the authors, Scaling-LAW-based MetaModels (SLAWMM) [17], where only aspect ratios of the studied component are varied, this paper extends the modeling to cases where other physical variables may also be varied.

The models derived in the paper give the expression of the convective heat transfer coefficient for a Limited Angle Torque Motor (LATM) when it is cooled by the surrounding air or by a fluid passing through its air gap. The obtained expressions are simple enough to be easily handled in preliminary design stage, yet predicting with sufficient accuracy outside the Design of Experiments (DoE) range where they where constructed.

Finally, an example is given to illustrate the SLAWMM use for the selection and preliminary design of a LATM with its cooling strategy. The most important outcome of the obtained models is that they permit to the designer to have rapid rough estimations of the design when comparing several system architectures.

\section{Acknowledgements}

This work has been partly funded by the European ACTUATION 2015 project (Modular Electro Mechanical Actuators for ACARE 2020 aircraft and helicopters) and by the French SYRENA project (SYstme de REgulation Nouvelles Architectures).

\section{Appendix A.}

This section illustrates how the convective heat transfer coefficient of a cylindrical shape, expressed and calculated in terms of heat flux rate density, may be expressed in terms of temperature difference. The general expression of the convective heat transfer coefficient, obtained in section 4.2 , is

$$
h=A \pi_{2}^{B} G r^{C} \lambda L^{-1}
$$

where $\pi_{2}=\frac{D}{L}$ and $G r=\frac{\rho^{2} g \beta \varphi L^{4}}{\mu^{2} \lambda}$. As it can be noted, it depends on the heat flow rate density $\varphi$. According to the Newton law:

$$
Q=\Delta \theta h S
$$

where $Q$ is the heat flux to be dissipated and $S$ the motor external surface. Equation (A.2) can also be expressed as:

$$
\frac{Q}{S}=\varphi=\Delta \theta h
$$

By replacing $\varphi$ from eq. (A.3) in the Grashof number and in eq. (A.1) gives:

$$
h=\underbrace{A\left(\frac{\rho^{2} g \beta}{\mu^{2} \lambda}\right)^{C}}_{k} \lambda\left(\frac{D}{L}\right)^{B}\left(\Delta \theta h L^{4}\right)^{C} L^{-1}=k D^{B} L^{4 C-1-B} \Delta \theta^{C} h^{C}
$$

Thus, the convective heat transfer coefficient can be expressed from eq. (A.4) as:

$$
h=k^{\frac{1}{1-C}} D^{\frac{B}{1-C}} L^{\frac{4 C-1-B}{1-C}} \Delta \theta^{\frac{C}{1-C}}
$$

\section{Appendix B.}

This section illustrates how are obtained the dimensionless numbers in eqs. (3) and (6). These numbers can be obtained by several methods; we used the method described in [25].

The procedure which helps to obtain the dimensionless numbers is based on the use of a table where are listed the variables (quantities) that may construct the dimensionless number, along with their dimensions (usually expressed in SI base units: length, mass, time, electric current, temperature, amount of substance and luminous intensity). Thus, for the construction of the dimensionless number from eq. (3), table B.2 can be constructed. In the first part of this table are listed the physical variables involved in the problem, with their associated dimensions. For instance, the dimension of the torque is $N m=k g \frac{m}{s^{2}} m=k g m^{2} s^{-2}$. The exponent of each dimension is noted in the corresponding column. This step is repeated for every variable. Since dimensionless numbers don't have dimensions, the idea is to find how can be combined the physical variables (divided or multiplied) so that the sum of the exponents for each dimension gives zero. These combinations are presented in the second part of the table B.2. We can see that in order to cancel the $\mathrm{kg}$ from the torque, only the induction of the permanent magnet can be used. Thus the first group that can be formed is $\frac{T}{B_{r}}$, which has the dimension $m^{2} A$. In order to cancel the $A$ from the first group only current density can be used. The second group is then $\frac{T}{B_{r} J}$, whose dimension is $m^{4}$. Finally, in order to cancel the $m$ from the second group we can use the length at forth power, which gives $\frac{T}{B_{r} J L^{4}}$. All the dimensions have been canceled, so the last obtained group is a valid dimensionless number. Exactly the same procedure is followed in table B.3 to obtain the dimensionless number from the eq. (6). 
Table B.2: Construction of the dimensionless number from eq. (3)

\begin{tabular}{lccccc}
\hline Quantity & Symbol & \multicolumn{4}{c}{ Dimensions } \\
& & $k g$ & $m$ & $s$ & $A$ \\
\hline Torque & $T$ & 1 & 2 & -2 & 0 \\
Length & $L$ & 0 & 1 & 0 & 0 \\
Current density & $J$ & 0 & -2 & 0 & 1 \\
Induction of PM & $B_{r}$ & 1 & 0 & -2 & -1 \\
\hline & $\frac{T}{B_{r}}$ & 0 & 2 & 0 & 1 \\
& $\frac{T}{B_{r} J}$ & 0 & 4 & 0 & 0 \\
& $\frac{T}{B_{r} J L^{4}}$ & 0 & 0 & 0 & 0 \\
\hline
\end{tabular}

Table B.3: Construction of the dimensionless number from eq. (6)

\begin{tabular}{lcccccc}
\hline Quantity & Symbol & \multicolumn{5}{c}{ Dimensions } \\
& & $k g$ & $m$ & $s$ & $K$ & $A$ \\
\hline Current density & $J$ & 0 & -2 & 0 & 0 & 1 \\
Length & $L$ & 0 & 1 & 0 & 0 & 0 \\
Electric resistivity & $\rho_{e l}$ & 1 & 3 & -3 & 0 & -2 \\
Temperature & $\theta$ & 0 & 0 & 0 & 1 & 0 \\
heat transfer coefficient & $h$ & 1 & 0 & -3 & -1 & 0 \\
\hline & $\frac{\rho_{e l}}{h}$ & 0 & 3 & 0 & 1 & -2 \\
& $\frac{\rho_{e l} J^{2}}{h}$ & 0 & -1 & 0 & 1 & 0 \\
& $\frac{\rho_{e l} J^{2}}{h \theta}$ & 0 & -1 & 0 & 0 & 0 \\
& $\frac{\rho_{e l} J^{2} L}{h \theta}$ & 0 & 0 & 0 & 0 & 0 \\
\hline
\end{tabular}

\section{References}

[1] Rosero J, Ortega J, Aldabas E, Romeral L. Moving towards a more electric aircraft. IEEE Aerospace and Electronic Systems Magazine 2007;22(3).

[2] Hendershot JR, Miller TJE. Design of Brushless Permanent-magnet Motors. Oxford University Press; 1995.

[3] Long G. Future directions in aeronautical composites. In: 21st ICAS Congress. Melbourne, Australia; 1998, p. 1-19.

[4] Gilson E, John DK, David M. Moog next generation control and actuation. In: Maré JC, editor. Recent Advances in Aerospace Actuation Systems and Components. Toulouse; 2014, p. 43-54.

[5] Descamps D, Alexandre P, Telteu-Nedelcu D. Hi-rel electromechanical thrust vector actuation systems for european unmanned launch vehicles a challenge for the next generation. In: Maré JC, editor. Recent Advances in Aerospace Actuation Systems and Components. Toulouse; 2012, p. 11-5

[6] Auweraer H, Anthonis J, Bruyne S, Leuridan J. Virtual engineering at work: the challenges for designing mechatronic products. Engineering with Computers 2013;29(3):177-667.

[7] Armaly BF, Chen TS, Ramachandran N. Correlations for laminar mixed convection on vertical, inclined and horizontal flat plates with uniform surface heat flux. International Journal of Heat and Mass Transfer 1987;30(2):405-8.

[8] Churchill SW, Ozoe H. A correlation for laminar free convection from a vertical plate. Journal of Heat Transfer $1973 ;: 540-1$.

[9] Churchill SW, Chu HHS. Correlating equations for laminar and turbulent free convection from a horizontal cylinder. International Journal of Heat and Mass Transfer 1975;18(9):1049-53.

[10] Minkowycz WJ, Sparrow EM. Local non similar solutions for natural convection on a vertical cylinder. Journal of Heat Transfer 1974;:178-83.

[11] Rohsenow W, Hartnett J, Cho Y. Handbook of heat transfer. New York: McGraw-Hill; 3 ed.; 1998.

[12] Yovanovich MM. On the Effect of Shape, Aspect Ratio and Orientation upon natural Convection from Isothermal Bodies of Complex Shape. In: ASME Winter Annual Meeting. Boston; 1987, p. 121-9.

[13] Palomo Del Barrio E, Lefebvre G, Behar P, Bailly N. Using model size reduction techniques for thermal control applications in buildings. Energy and Buildings 2000;33(1):1-14.

[14] Raymond HM, Montgomery DC, Christine MAC. Response surface methodology : process and product optimization using designed experiments. John Wiley \& Sons; 4th ed.; 2006.

[15] Forrester A, Sóbester A, Keane AJ. Engineering design via surrogate modelling: a practical guide. J. Wiley; 2008.

[16] Montgomery DC. Design and Analysis of Experiments. Eighth edi ed.; 2012. ISBN 9781118146927.

[17] Budinger M, Passieux JC, Gogu C, Fraj A. Scaling-law-based metamodels for the sizing of mechatronic systems. Mechatronics in press;doi: 10.1016/j.mechatronics.2013.11.012.

[18] West GB. The origin of allometric scaling laws in biology from genomes to ecosystems: towards a quantitative unifying theory of biological structure and organization. Journal of Experimental Biology 2005;208(9):1575-92.

[19] Budinger M, Liscouët J, Hospital F, Maré JC. Estimation Models for the Preliminary Design of Electro-Mechanical Actuators. Proceedings of the Institution of Mechanical Engineers, Part G: Journal of Aerospace Engineering 2012;3:243-59.

[20] Mendez PF, Ordonez F. Scaling Laws From Statistical Data and Dimensional Analysis. Journal of Applied Mechanics 2005;72(5):648-57.

[21] Pahl G, Beitz W, Feldhusen J, Grote KH. Engineering Design: A Systematic Approach. Springer; 3 ed.; 2007.

[22] Petersheim MD, Brennan SN. Scaling of hybrid-electric vehicle powertrain components for Hardware-in-the-loop simulation. Mechatronics 2009;19(7):1078-90.

[23] Miragliotta G. The power of dimensional analysis in production systems design. International Journal of Production Economics 2011;131(1):175 -82 .

[24] Murphy G. Similitude in engineering. New York: The Ronald press company; 1950.

[25] Taylor ES. Dimensional Analysis for engineers. Oxford University Press; 1974.

[26] Parvex . NX series Motor Datasheet. ???? URL http://www.parvex.com/english/products/datasheet_nx.htm.

[27] Kollmorgen . Goldline B/M \& BH/MH Series. ???? URL http://www.kollmorgen.com/en-us/products/motors/servo/goldline-

[28] Myers RH, Montgomery DC, Anderson-Cook CM. Response surface methodology: process and product optimization using designed experiments. Wiley; 3 ed.; 2009.

[29] Jin R, Chen W, Simpson TW. Comparative studies of metamodeling techniques under multiple modeling criteria. Structural and Multidisciplinary Optimization 2000;23:1-13.

[30] Simpson T, Poplinski J, Koch PN, Allen J. Metamodels for computerbased engineering design: survey and recommendations. Engineering with Computers 2001;17(2):129-50.

[31] Kleijnen JP. Kriging metamodeling in simulation: A review. European Journal of Operational Research 2009;192(3):707 -16.

[32] Stein ML. Interpolation of spatial data: some theory for kriging. Springer; 1999.

[33] Bencheikh N, Letty RL, Claeyssen F, Magnac G, Migliorero G. Limited angel torque actuator for fine angular positioning. In: Proceedings of the 12th International Conference on New Actuators. Bremen, Germany; 2010 .

[34] Tsai CC, Lin SC, Huang HC, Cheng YM. Design and control of a brushless DC limited-angle torque motor with its application to fuel control of smallscale gas turbine engines. Mechatronics 2009;19(1):29-41.

[35] FHL . Excellence in aerospace actuation and support chain management. 2002. doi:ED04-6/02.

[36] Cebeci T, Bradshaw P. Physical and Computational Aspects of Convective Heat Transfer. Berlin: Springer-Verlag Berlin Heidelberg; 1984. doi: 10.1007/978-3-662-02411-9.

[37] Laïd M. Extraction de modèles thermiques simplifiés des machines électriques à partir dun calcul du champ de températures. Ph.D. thesis; Université Paris-Sud; 2011.

[38] Kollmorgen . Limited Angle Torquers (LATs). 2010. doi: AD3MBHBB0598-1017.

[39] Roos F, Johansson H, Wikander J. Optimal selection of motor and gearhead in mechatronic applications. Mechatronics 2006;16(1):63-72.

[40] Budinger M, Liscouet J, Hospital F, Mare JC. Estimation models for the preliminary design of electromechanical actuators. Proceedings of 
the Institution of Mechanical Engineers, Part G: Journal of Aerospace Engineering 2011;226(3):243-59. 